\title{
Mapping evidence of interventions and strategies to bridge the gap in the implementation of the prevention of mother-to-child transmission of HIV programme policy in sub-Saharan countries: A scoping review
}

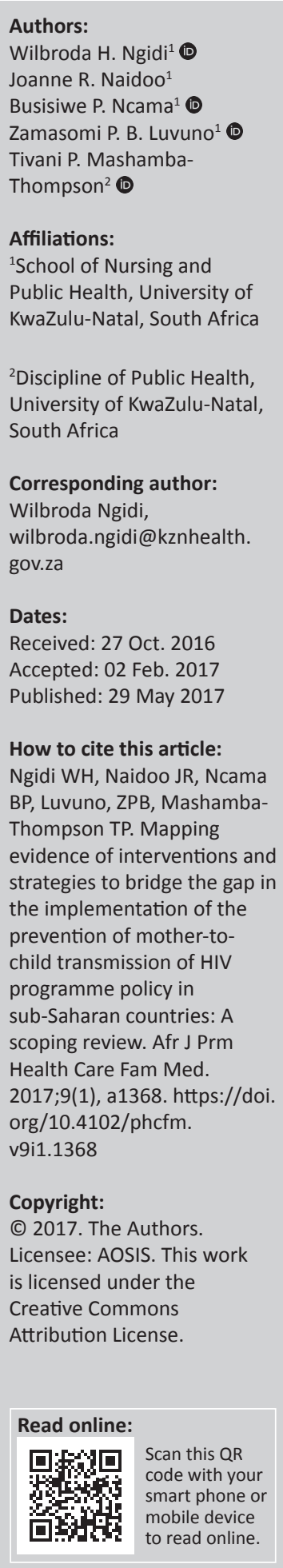

Background: Prevention of mother-to-child transmission (PMTCT) of HIV is a life-saving public health intervention. Sub-Saharan African (SSA) countries have made significant progress in the programme, but little is known about the strategies used by them to eliminate mother-to-child transmission of HIV.

Aim: To map evidence of strategies and interventions employed by SSA in bridging the implementation gap in the rapidly changing PMTCT of HIV programme policy.

Methods: Electronic search of the databases MEDLINE, PubMed and SABINET for articles published in English between 2001 and August 2016. Key words included 'Sub-Saharan African countries', 'implementation strategies', 'interventions to bridge implementation gap', 'prevention of mother-to-child transmission of HIV' and 'closing implementation gap'.

Results: Of a total of 743 articles, 25 articles that met the inclusion criteria were included in the study. Manual content analysis resulted in the identification of three categories of strategies: (1) health system (referral systems, integration of services, supportive leadership, systematic quality-improvement approaches that vigorously monitors programme performance); (2) health service delivery (task shifting, networking, shared platform for learning, local capacity building, supportive supervision); as well as (3) community-level strategies (community health workers, technology use - mHealth, family-centred approaches, male involvement, culturally appropriate interventions).

Conclusion: There are strategies that exist in SSA countries. Future research should examine multifaceted scientific models to prioritise the highest impact and be evaluated for effectiveness and efficiency.

\section{Introduction}

Sub-Saharan African (SSA) countries are highly burdened by HIV. ${ }^{1}$ The children are at risk of transmission of HIV from mother-to-child, which is a major public health concern. The programme 'prevention of mother-to-child transmission (PMTCT) of HIV' is aimed at addressing this problem. It has been demonstrated that the risk of mother-to-child transmission (MTCT) of HIV can be reduced by implementing key interventions, especially in resource limited settings. Countries are working towards ${ }^{2,3}$ meeting the Global Plan targets of the elimination of MTCT of HIV. Also, eradicating the HIV epidemic by 2020 remains the key target of the Sustainable Development Goal on health and well-being. ${ }^{1,3,4,5}$

Sub-Saharan African countries have demonstrated significant progress in the scaling up of PMTCT. The number of new HIV infections among children had significantly dropped from 350000 in 2009 to less than 199000 by 2013, which is a 43\% decline in SSA countries. However, this is not in tune with the $90 \%$ reduction aimed for in the Global Plan (2011-2015). ${ }^{4}$ Disparities exist in the reduction of new HIV infection in children in the SSA countries. It is clear that the progress is slow, and efforts to accelerate progress are needed for countries to achieve the universal targets of $90-90-90 \cdot^{1,3,4,5,6}$

Much evidence exists demonstrating barriers to PMTCT programme policy implementation, $2,7,8,9,10,11,12,13$ meant to save lives. While World Health Organization (WHO) had recommendations for countries to adopt including Option B+ which promotes universal life-long 
antiretroviral treatment (ART) for all, crucial barriers to successful implementation remain..$^{4,9,14,15}$ Strategies are employed by the SSA countries to bridge the implementation gap for the PMTCT programme. As appreciation of the impact of implementation of policy develops interest, it is crucial that the evidence of such interventions is identified and mapped out.

The aim of the scoping was to map the existing literature on the interventions and strategies applied in the SSA countries to close PMTCT of HIV policy implementation.

\section{Methods \\ Search strategy}

The scoping review involved a comprehensive systematic literature search in MEDLINE, PubMed, SABINET databases, including the reference list of included studies and key websites from WHO and UNAIDS. Two independent reviewers (WH and ZL) screened all titles and abstracts using predetermined inclusion and exclusion criteria, reviewed full text articles for inclusion, with disagreements settled by third and fourth reviewers (BN, JN) to ensure reliability of findings. The keyword search included 'sub-Saharan African countries, implementation gap strategies, interventions to bridge implementation gap, prevention of mother-to-child transmission of $\mathrm{HIV}$, closing implementation gap for PMTCT'.

The search was limited to English language publications between 2001 and August 2016. Studies were included if they reported on interventions or strategies for bridging implementation gap for PMTCT of HIV health programme policy in SSA countries; if they were fully accessible; and if they dealt with public health setting strategies or communitylevel interventions.

We excluded studies that were not published in English; not conducted in SSA countries, did not provide the setting; did not provide strategies; focused their intervention on programmes other than PMTCT; had inaccessible full texts; had inappropriate methodology; had comments or debates; were published prior to 2001 .

\section{Data extraction and analysis}

The search criteria used PRISMA 2009 diagram tool ${ }^{16}$ to map relevant searched articles (see Figure 2). A standardised data extraction form was used to guide eligibility of extracted studies edited from PICOS framework (Population, Intervention, Comparison, Outcome, Study design) which charted information on author and year, study design, population, $\%$ of population, intervention, aim of the study as well as the key findings including recommendations if any (see Table 1). The review was not limited to study designs. Manual content analysis was used to identify key categories and reporting on key findings. Mixed Method Appraisal Tool (MMAT) was used for quality assessment of included studies. ${ }^{17,18}$

\section{Ethical consideration}

This article is part of a PhD thesis, which is being currently conducted in accordance with permission from the ethics committee of the University of KwaZulu-Natal (UKZN) BREC (Biomedical Research Ethics Council), under the protocol reference number BE112/14.

\section{Review results}

The three-step study selection process for this scoping review comprised (1) first step: database search using keywords and title screening that returned 734 articles; 11 additional records were identified through reference lists and websites for WHO and UNAIDS. A total of 329 records after exclusion criteria, duplicates and non-English records were excluded; (2) second step: screening of titles and abstracts identified 73 records for full text review after exclusion of 256 papers which were either not conducted in SSA countries or reported on strategies or interventions for other health programmes and not for the PMTCT programme; (3) third step was full article screening, done in duplicate on 73 records, which resulted in 44 records being excluded for providing insufficient information; no description of interventions or strategies of interest; the full article not being accessible; for being in comment or debate form of report and conducted prior to 2001. This resulted in 29 records assessed for quality using MMAT tool checklist; however, a total of four articles were excluded for reasons such as inappropriate methodology or for not providing key findings (report on a protocol for randomised control trial). Average time spent critically appraising one article was 15 minutes. At the end of the final review and assessment, 25 eligible studies were included for final analysis. Details related to the search are provided in the PRISMA flow diagram (see Table 1). ${ }^{19,20,21}$

Papers were mainly from six specific countries as well as from studies done across SSA countries as follows: South Africa $(n=8)$; Nigeria $(n=2)$; Uganda $(n=2)$; Malawi $(n=3)$; and a study done in three different countries of Malawi, Nigeria and Zimbabwe $(n=1)$, including studies done in SSA countries $(n=9)$ not specifically in one country. Majority of studies conducted in SSA countries were systematic reviews $(n=9)$, studies reporting on operational research $(n=3)$, qualitative studies $(n=6)$, quantitative studies $(n=4)$ and record review studies $(n=3)$. There were neither randomised controlled trial studies nor studies of mixed methods.

\section{Types of strategies}

Studies reported on the various stages of the implementation of PMTCT of HIV programmes by SSA countries ranging from the use of Option B to that of Option B+ as recommended by WHO. ${ }^{3,6}$

After content analysis, the three most significant strategies and interventions that were in line with our objectives and inclusion criteria were categorised as follows: health service delivery, community-based interventions and health system strategies (see Figure 1). 


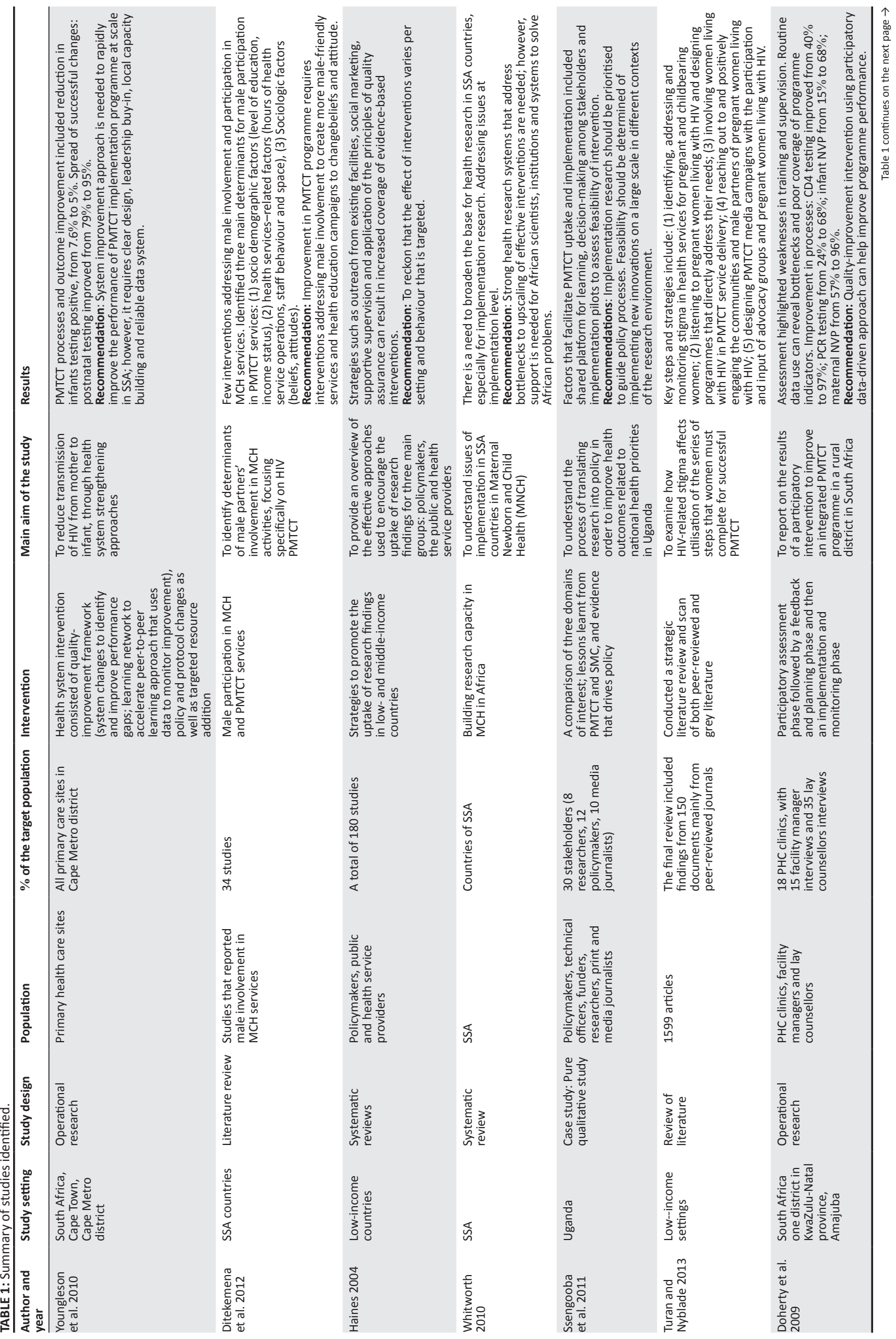




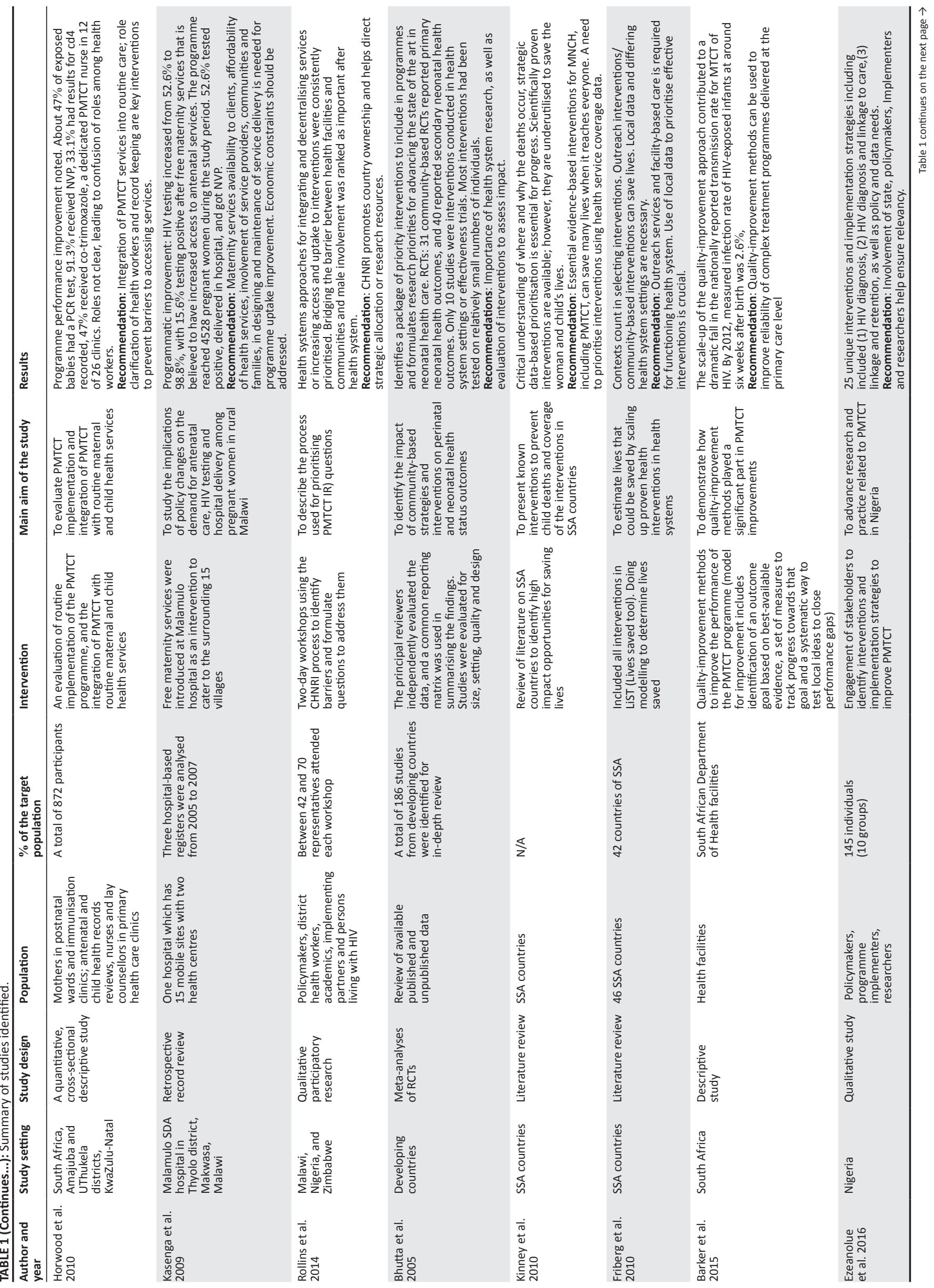




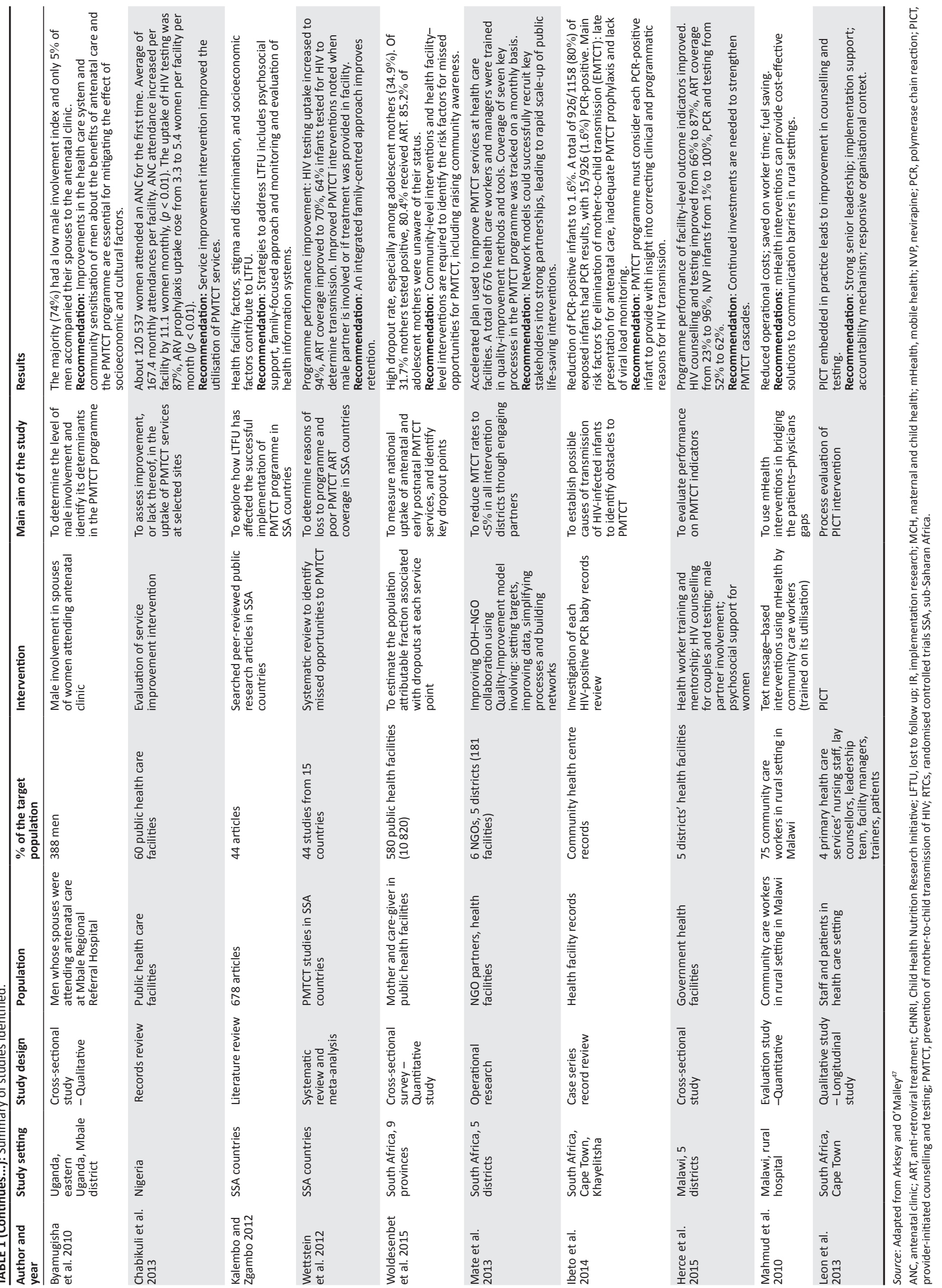




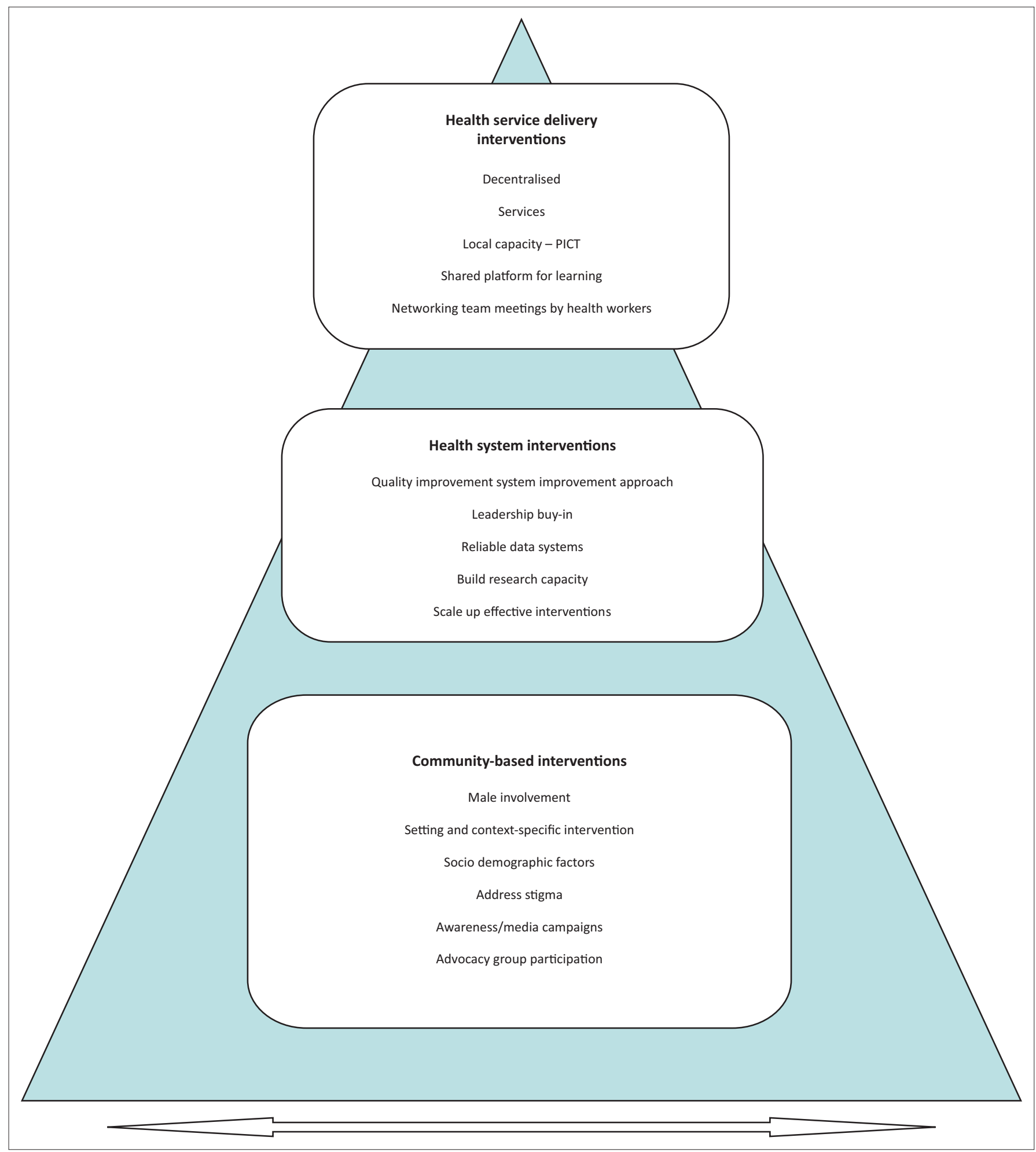

FIGURE 1: Model developed providing summary of three key categories from final articles.

\section{Health service delivery strategies}

The common strategies reported included shared platform for learning and decision-making among stakeholders, ${ }^{22}$ health service improvement of processes ${ }^{2,23,24}$ and data-driven qualityimprovement approach which was mainly reported in South Africa. $25,26,27,28$ This approach included health workers forming information teams to discuss issues relating to performance, forming learning networks which also promote shared learning, and discussing changes and planning for implementation.
There were studies that reported on strengthening of integration of PMTCT services into maternal health programme as well as clear role clarifications among the implementers $8,10,29,30$; reinforcement of provider-initiated counselling and testing services by health workers to improve uptake ${ }^{31}$ which is also linked to task shifting. Another approach for assisting in bridging the gap of PMTCT implementation is monitoring individual infants to determine the reason for transmission so that interventions can be targeted at 


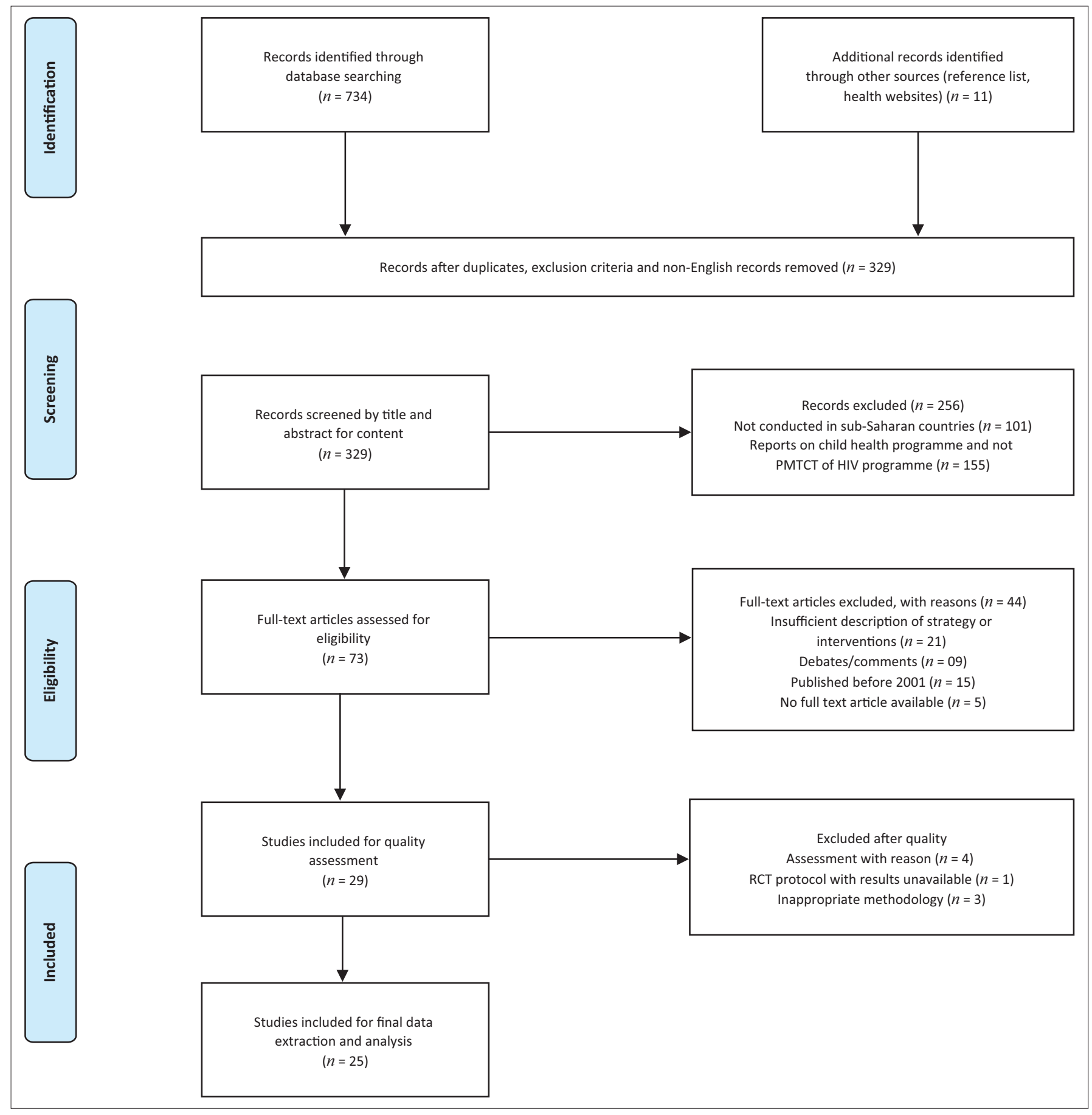

Source: Moher et al ${ }^{46}$

FIGURE 2: PRISMA 2009 flow diagram of searched articles.

the barriers. ${ }^{32}$ This, according to the study, can reveal specific barriers to be addressed for countries to get to zero infections.

\section{Community-based strategies}

It was common among assessed articles that the consideration of context and settings remained crucial, especially in SSA countries. ${ }^{11,12,33,34}$ The reality on the ground is seen as one of the major barriers to implementation of prevention programmes such as the PMTCT programme; hence, strategies should be tailor-made to cater to such barriers. The importance of community involvement in bridging the gap in policies remains crucial. ${ }^{21,35,36,37}$
There were articles that considered the promotion of disclosure of HIV infection in ensuring that communities are supported in the programme. ${ }^{12}$ According to health care workers, failure to disclose can result in poor support. Owing to the fact that the programme needs communities to take part for its success, linkage between public health facilities and communities was seen as one strategy to close gaps in implementation.

Some models included the community care-givers, strengthened the linkages and the referral system between communities and health facilities ${ }^{37,38,39}$ as well as made 
communities aware through community sensitisation models, including conducting campaigns in communities to create awareness and to address stigma which is reported as a barrier in communities. ${ }^{34,37,40}$

In the inclusion of families using family-centred approach ${ }^{41}$, male involvement ${ }^{36,38}$ was identified as a gap where only women seem to be the focus. This calls for more engagement of men in future studies. For some studies, innovations, including use of technology, for example use of text messages by community care workers (mHealth) to reach communities, ${ }^{39}$ demonstrate how technology can be utilised to access health.

Access to services remains crucial for any prevention programme to be a success; therefore, free and affordable services that can improve uptake of services are employed ${ }^{41}$; however, considering contexts, the affordability of such interventions across countries and sustainability of such an approach need to be further explored.

\section{Health system interventions}

Health system-related interventions included among others the importance of leaders in the health system to pave the direction for implementers; hence, leadership buy-in ${ }^{27}$ to effect change in the use of quality-improvement model for improvement is proposed for the implementation to take place. However, models on leadership engagement need to be explored for countries to maximise benefits from it.

The reviews highlighted the importance of using reliable data systems with the health workers as implementers and adopting an approach that addresses health system improvement ${ }^{25,26,27,28}$ as one of the strategies, for example the use of quality-improvement approaches towards improvement that promotes health system strengthening. $\mathrm{WHO}$, in its framework of six building blocks, highlighted information as one of the health system-building blocks and the importance of monitoring health system performance of programmes for progress as well as impact. ${ }^{23,42}$

Strengthening research capacity among SSA countries is seen as vital in improving implementation. This included capacity building on implementation science research targeting health workforce, especially in resource limited countries. ${ }^{4,27,43,44,45}$ By doing so, it is believed that low-resource countries can better understand implementation science as it relates to answering critical research questions in their context.

Figure 1 represents the model to summarise identified key categories.

\section{Discussion}

Our review on mapping strategies and interventions to bridge the gap in the implementation of PMTCT of HIV in the SSA countries had found articles on strategies and promising approaches used while identifying areas for future research. Based on this review, majority of papers are secondary papers $(n=9)$, reporting on systematic reviews and lack primary researches reported on this area. Papers also reported on barriers to implementation and enablers thereof with limited studies reporting on strategies for closing the implementation gap. It was common that for a strategy to be effective, it needed to be informed by identified barriers to implementation. ${ }^{34}$

Evidence regarding particular strategies mostly relates to the PMTCT programme performance through data indicators monitoring of the PMTC cascade. Previous evidence had mainly focused on monitoring performance for PMTCT indicators through the PMTCT cascade. This was used to measure programme implementation, and identified gaps in the PMTCT programme coverage which identified strategies that included using quality-improvement approaches in public health care settings that focus on obtaining and building will from leadership, ideas for changes, monitoring data as well as using platforms for shared learning which was common across studies. $2,25,26,27,32,48$

While task shifting emerged as one of the key solutions to bridge policy implementation gaps, and was seen as a strategy to improve healthcare coverage as well as address human resources challenges which were common across the SSA countries, inadequate supervision and poor role clarification remain a challenge..$^{8,49}$

Majority of papers report on health worker interventions including health system intervention, ${ }^{22,25,26,27}$ with the majority reporting on the importance of engaging implementers for any implementation gap to be closed ${ }^{26,27,31,39,43,44}$ which was found to be crucial, especially in low-resource countries.

While the strategies were offered by the SSA countries, they lack evaluations to determine the effectiveness of those identified strategies. ${ }^{44}$ The lack of evaluation of implementation strategies hinders their assessment for effectiveness. Interventions reported including free service delivery ${ }^{41}$ may require further assessment for sustainability of such an approach in a given low-resource country; however, the majority of SSA countries were offering free maternity services.

With countries expected to eliminate MTCT of HIV, demand for countries to speed up implementation and package interventions and strategies that are effective in closing the implementation gap remain crucial.

\section{Strengths and limitations of this study}

The review was limited to studies published in English, as it is the commonly used language for communication in the majority of SSA countries. The review was limited to articles published from 2001 to August 2016, and publications prior to this year were excluded for relevancy of the research topic as well as for reasons related to PMTCT not fully being implemented in SSA countries before 2001. The review focused on articles published in SSA countries, due to comparable settings and similar resources available. 
Our review focused on the strategies and interventions for PMTCT of HIV programme to close the gap in implementation, and therefore excluded the broader aspect of other programmes that may offer strategies to close the gap in implementation; however, this review can guide future research so that a broader evidence of effective strategies used in health and other prevention programmes can be mapped.

\section{Conclusions and recommendations}

Prevention of MTCT of HIV remains a growing global public health priority, more so in the highly HIV-burdened SSA countries. This review revealed that there is no one size fits all approach in closing the implementation gap for priority prevention programmes such as PMTCT of HIV in countries with low resources. There is a need for a combination of multifaceted approaches that consider contextual settings, leadership involvement, shared learning forums while considering resources towards closing the implementation gap.

Packaging of strategies that have proven to work for scale-up to similar settings is needed; however, evaluation of such interventions and strategies for effectiveness and efficiency is of vital importance. The Global Plan vision (2011-2015), of no child shall be born with HIV and keeping mothers alive as well as eliminating MTCT of HIV until it does not constitute a public health concern, as well as achievement of universal 90-90-90 strategies require unusual business approaches as well as the engagement with researchers, policymakers, implementers and communities to prioritise implementation in the policy development phase, considering various contextual settings.

\section{Acknowledgements}

The reviewers appreciate the School of Nursing support throughout the review process.

\section{Competing interests}

The authors declare that they have no competing interests.

\section{Authors' contributions}

W.H.N. was responsible for the conceptualisation of the review. T.M. assisted in the design of the article. W.H. and Z.L. devised the plan for data extraction. All authors critically reviewed and revised the manuscript. B.P. and J.N. were the third and the fourth reviewers for the confirmation of disagreements. W.H.N. prepared the final manuscript. All authors were involved in the final manuscript review and approval.

\section{References}

1. UNAIDS. Global plan towards the elimination of new HIV infections among children by 2015. Geneva, Switzerland: UNAIDS; 2014.

2. Herce ME, Mtande T, Chimbwandira F, et al. Supporting Option B+ scale up and strengthening the prevention of mother-to-child transmission cascade in central Malawi: Results from a serial cross-sectional study. BMC Infec Dis. 2015;15(1):1. $\mathrm{http}: / /$ dx.doi.org/10.1186/s12879-015-1065-y
3. UNAIDS. Global AIDS update 2016. Geneva, Switzerland: UNAIDS; 2016.

4. World Health Organization. Global update on HIV treatment 2013: Results, impact and opportunities. Geneva, Switzerland: WHO; 2013.

5. Sidibé M, Loures L, Samb B. The UNAIDS 90-90-90 target: A clear choice for ending AIDS and for sustainable health and development. J Int AIDS Soc. 2016;19(1):21133. http://dx.doi.org/10.7448/IAS.19.1.21133

6. WHO. Use of antiretroviral drugs for treating pregnant women and preventing HIV infection in infants. Geneva: WHO; 2012.

7. Chopra M, Daviaud E, Pattinson R, Fonn S, Lawn JE. Saving the lives of South Africa's mothers, babies, and children: Can the health system deliver? South Africa. Lancet. 2009;374(9692):835-846. http://dx.doi.org/10.1016/S0140-6736(09)61123-5

8. Kinney MV, Kerber KJ, Black RE, et al. Sub-Saharan Africa's mothers, newborns, and children: Where and why do they die? PLoS Med. 2010;7(6):e1000294. $\mathrm{http}: / / \mathrm{dx}$.doi.org/10.1371/journal.pmed.1000294

9. Peltzer K, Mosala T, Shisana O, Nqueko A, Mngqundaniso N. Barriers to prevention of HIV transmission from mother to child (PMTCT) in a resource poor setting in the Eastern Cape, South Africa. Afr J Reprod Health. 2007;11(1):57-66. http://dx.doi. org/10.2307/30032488

10. Stringer EM, Sinkala M, Stringer JS, et al. Prevention of mother-to-child transmission of HIV in Africa: Successes and challenges in scaling-up a nevirapinebased program in Lusaka, Zambia. AIDS (London, England). 2003;17(9):1377. http://dx.doi.org/10.1097/00002030-200306130-00012

11. Aizire J, Fowler MG, M Coovadia H. Operational issues and barriers to implementation of prevention of mother-to-child transmission of HIV (PMTCT) interventions in Sub-Saharan Africa. Curr HIV Res. 2013;11(2):144-159. http:// dx.doi.org/10.2174/1570162X11311020007

12. Nuwagaba-Biribonwoha $H$, Mayon-White R, Okong P, Carpenter L. Challenges faced by health workers in implementing the prevention of mother-to-child HIV transmission (PMTCT) programme in Uganda. J Public Health. 2007;29(3): 269-274. http://dx.doi.org/10.1093/pubmed/fdm025

13. Sherman G, Jones $S$, Coovadia A, Urban M, Bolton K. PMTCT from research to reality-results from a routine service. S Afr Med J. 2004;94(4):289-292.

14. Both J, Van Roosmalen J. The impact of Prevention of Mother to Child Transmission (PMTCT) programmes on maternal health care in resource-poor settings: Looking beyond the PMTCT programme - A systematic review. Brit J Obstet Gynaec 2010;117(12):1444-1450. http://dx.doi.org/10.1111/j.1471-0528.2010.02692.x

15. Doherty TM, McCoy D, Donohue $S$. Health system constraints to optimal coverage of the prevention of mother-to-child HIV transmission programme in South Africa: Lessons from the implementation of the national pilot programme. African Health Sci. 2005;5(3):213-218.

16. Moher D, Liberati A, Tetzlaff J, Altman DG. Preferred reporting items for systematic reviews and meta-analyses: The PRISMA statement. Ann Intern Med. 2009; 151(4):264-269. http://dx.doi.org/10.7326/0003-4819-151-4-200908180-00135

17. Pace R, Pluye $P$, Bartlett $G$, et al. Testing the reliability and efficiency of the pilot Mixed Methods Appraisal Tool (MMAT) for systematic mixed studies review. Int Nurs Stud. 2012;49(1):47-53. http://dx.doi.org/10.1016/j.ijnurstu.2011.07.002

18. Pluye P. Critical appraisal tools for assessing the methodological quality of qualitative, quantitative and mixed methods studies included in systematic mixed studies reviews. J Eval Clin Pract. 2013;19(4):722. http://dx.doi.org/10.1111/ jep.12017

19. Friberg IK, Kinney MV, Lawn JE, et al. Sub-Saharan Africa's mothers, newborns, and children: How many lives could be saved with targeted health interventions? PLoS Med. 2010;7:e1000295. http://dx.doi.org/10.1371/journal.pmed.1000295

20. Turan JM, Nyblade L. HIV-related stigma as a barrier to achievement of global PMTCT and maternal health goals: A review of the evidence. AIDS Behav. 2013;17:2528-2539. http://dx.doi.org/10.1007/s10461-013-0446-8

21. Woldesenbet $S$, Jackson $D$, Lombard $C$, et al. Missed opportunities along the prevention of mother-to-child transmission services cascade in South Africa: Uptake, determinants, and attributable risk (the SAPMTCTE). PLoS One. 2015;10:e0132425. http://dx.doi.org/10.1371/journal.pone.0132425

22. Ssengooba F, Atuyambe L, Kiwanuka SN, Puvanachandra P, Glass N, Hyder AA. Research translation to inform national health policies: Learning from multiple perspectives in Uganda. BMC. 2011;11(1):1. http://dx.doi.org/10.1186/1472 698x-11-s1-s13

23. Chabikuli O, Gwarzo U, Olufunso A, et al. Closing the prevention of mother-tochild transmission gap in Nigeria: An evaluation of service improvement intervention in Nigeria. S Afr Fam Pract. 2013;55(1):96-102. http://dx.doi.org/10 1080/20786204.2013.10874310

24. Ezeanolue EE, Powell BJ, Patel D, et al. Identifying and prioritizing implementation barriers, gaps, and strategies through the Nigeria implementation science alliance: Getting to zero in the prevention of mother-to-child transmission of HIV. J Acquir Immune Defic Syndr. 2016;72:S161-S166. http://dx.doi.org/10.1097/ QAl.0000000000001066

25. Doherty T, Chopra M, Nsibande D, Mngoma D. Improving the coverage of the PMTCT programme through a participatory quality improvement intervention in South Africa. BMC Pub Health. 2009;9(1):1. http://dx.doi.org/10.1186/14712458-9-406

26. Mate KS, Ngubane G, Barker PM. A quality improvement model for the rapid scale-up of a program to prevent mother-to-child HIV transmission in South Africa. Int J Qual Health Care. 2013;25:373-380. http://dx.doi.org/10.1093/intqhc/ mzt039

27. Youngleson MS, Nkurunziza P, Jennings K, Arendse J, Mate KS, Barker P. Improving a mother to child HIV transmission programme through health system redesign: Quality improvement, protocol adjustment and resource addition. PLoS One. 2010;5(11):e13891. http://dx.doi.org/10.1371/journal.pone.0013891 
28. Barker P, Barron P, Bhardwaj S, Pillay $Y$. The role of quality improvement in achieving effective large-scale prevention of mother-to-child transmission of HIV in South Africa. AIDS. 2015;29:S137-S143. http://dx.doi.org/10.1097/QAD. 0000000000000718

29. Horwood C, Haskins L, Vermaak K, Phakathi S, Subbaye R, Doherty T. Prevention of mother to child transmission of HIV (PMTCT) programme in KwaZulu-Natal, South Africa: An evaluation of PMTCT implementation and integration into routine maternal, child and women's health services. Trop Med Int Health. 2010;15(9): 992-999. http://dx.doi.org/10.1111/j.1365-3156.2010.02576.x

30. Welty TK, Bulterys M, Welty ER, et al. Integrating prevention of mother-to-child HIV transmission into routine antenatal care: The key to program expansion in Cameroon. J Acquir Immune Defic Syndr. 2005;40(4):486-493. http://dx.doi. org/10.1097/01.qai.0000163196.36199.89

31. Leon N, Lewin S, Mathews C. Implementing a provider-initiated testing and counselling (PITC) intervention in Cape Town, South Africa: A process evaluation using the normalisation process model. Implement Sci. 2013;8(1):1. http://dx. doi. org/10.1186/1748-5908-8-97

32. Ibeto M, Giddy J, Cox V. Closing the gaps: Steps towards elimination of mother-tochild transmission of HIV. Southern Afr J HIV Med. 2014;15(3):107-109. http:// dx.doi.org/10.7196/sajhivmed.1047

33. Buttenheim AM, Asch DA. Behavioral economics: The key to closing the gap on maternal, newborn and child survival for millennium development goals 4 and 5 ? Matern Child Health J. 2013;17(4):581-585. http://dx.doi.org/10.1007/s10995012-1042-7

34. Haines A. Bridging the implementation gap between knowledge and action for health. Bull World Health Organ. 2004;82(10):724-731.

35. Bhutta ZA, Darmstadt GL, Hasan BS, Haws RA. Community-based interventions for improving perinatal and neonatal health outcomes in developing countries: A review of the evidence. Pediatrics. 2005;115(Suppl 2):519-617. http://dx.doi org/10.1542/peds.2004-1441

36. Byamugisha R, Tumwine JK, Semiyaga N, Tylleskär T. Determinants of male involvement in the prevention of mother-to-child transmission of HIV programme in Eastern Uganda: A cross-sectional survey. Reprod Health. 2010;7(1):1. http:// dx.doi.org/10.1186/1742-4755-7-12

37. Tomlinson M, Doherty T, Jackson D, et al. An effectiveness study of an integrated, community-based package for maternal, newborn, child and HIV care in South Africa: Study protocol for a randomized controlled trial. Trials. 2011;12(1):1 http://dx.doi.org/10.1186/1745-6215-12-236
38. Ditekemena J, Koole O, Engmann C, et al. Determinants of male involvement in maternal and child health services in sub-Saharan Africa: A review. Reprod Health 2012;9(1):1. http://dx.doi.org/10.1186/1742-4755-9-32

39. Mahmud N, Rodriguez J, Nesbit J. A text message-based intervention to bridge the healthcare communication gap in the rural developing world. Technol Health Care. 2010;18(2):137-144.

40. Wettstein C, Mugglin C, Egger M, et al. Missed opportunities to prevent motherto-child-transmission in sub-Saharan Africa: Systematic review and meta-analysis. AIDS (London, England). 2012;26(18):2361. http://dx.doi.org/10.1097/QAD. Ob013e328359ab0c

41. Kasenga F, Byass $P$, Emmelin $M$, Hurtig A-K. The implications of policy changes on the uptake of a PMTCT programme in rural Malawi: First three years of experience. Global Health Action. 2009;2:1-7. http://dx.doi.org/10.3402/gha.v2i0.1883

42. World Health Organization. Monitoring the building blocks of health systems: A handbook of indicators and their measurement strategies. Geneva, Switzerland World Health Organization; 2010.

43. Rollins N, Chanza H, Chimbwandira F, et al. Prioritizing the PMTCT implementation research agenda in 3 African countries: INtegrating and Scaling up PMTCT through Implementation REsearch (INSPIRE). J Acquir Immune Defic Syndr. 2014; 67(Suppl 2):S108-S13. http://dx.doi.org/10.1097/QAI.0000000000000358

44. Whitworth J. Improving implementation: Building research capacity in maternal neonatal, and child health in Africa. PLoS Med. 2010;7(7):3. http://dx.doi.org/ 10.1371/journal.pmed.1000299

45. Williams C. Strategies for enhancing the implementation of school-based policies or practices targeting risk factors for chronic disease. Cochrane Database Syst Rev. 2015;5:1-21. http://dx.doi.org/10.1002/14651858.CD011677

46. Moher D, Liberati A, Tetzlaff J, Altman DG, Group P. Preferred reporting items for systematic reviews and meta-analyses: The PRISMA statement. PLoS Med. 2009b;6:e1000097. http://dx.doi.org/10.1136/bmj.b2535

47. Arksey H, O'Malley L. Scoping studies: Towards a methodological framework. International Journal of Social Research Methodology. 2005;8(1):19-32.

48. Kalembo FW, Zgambo M. Loss to followup: A major challenge to successfu implementation of prevention of mother-to-child transmission of HIV-1 programs in sub-Saharan Africa. Isrn AIDS. 2012;2012:1-10.

49. Crowley T, Mayers P. Trends in task shifting in HIV treatment in Africa: Effectiveness, challenges and acceptability to the health professions. Afr J Prim Health Care Fam Med. 2015;7(1):1-9. http://dx.doi.org/10.4102/phcfm.v7i1.807 